\title{
Análise dos encaminhamentos realizados pela triagem quanto ao acesso às clínicas do curso de Odontologia da Universidade Federal de Santa Maria
}

Juliane Bortolotto da Rosa*; Karine Andréia Barkert*; Gabriele Rissotto Menegazzo**; Silvia Ataide Pithan***; Jessye Melgarejo do Amaral Giordani****

* Cirurgiã-dentista, egressa do Curso de Odontologia Universidade Federal de Santa Maria

** Discente de Doutorado, Programa de Pós-Graduação em Ciências Odontológicas, Universidade Federal de Santa Maria

*** Professora Doutora, Curso de Odontologia, Universidade Federal de Santa Maria

**** Professor Doutor, Programa de Pós-Graduação em Ciências Odontológica, Curso de Odontologia, Universidade Federal de Santa Maria

Recebido em 04/04/2019. Aprovado em 12/02/2020.

\begin{abstract}
RESUMO
O objetivo deste estudo foi descrever o acesso ao tratamento e as necessidades terapêuticas dos pacientes que buscam atendimento nas clínicas do Curso de Odontologia da Universidade Federal de Santa Maria (Brasil) por meio dos encaminhamentos realizados pelo Serviço de Triagem do curso, bem como verificar quantos desses pacientes efetivamente receberam atendimento. Trata-se de um estudo observacional descritivo de caráter quantitativo, baseado em dados secundários existentes nos registros das listas de espera do Serviço de Triagem do curso e nos dados do software Gerenciador Odontológico usado para registro de procedimentos. Foram incluídos no estudo os dados dos pacientes que buscaram o serviço entre 2015 e 2017. Os dados foram analisados utilizando o programa estatístico STATA 14.0, apresentando frequências relativas e absolutas. O número total de encaminhamentos realizados no período foi de 3.945, a maioria destes para pessoas do sexo feminino e com idade média de 51,2 anos. Do total de encaminhamentos, em 60,2\% (2.377) dos casos o atendimento não se concretizou. Nas situações que resultaram em atendimento, em 77,6\% (1.221) dos casos, este ocorreu no mesmo ano do encaminhamento. A maioria dos encaminhamentos foram para clínicas de atendimento integrado, indicando um acúmulo de necessidades terapêuticas. Esse tipo de avaliação é fundamental para o planejamento estratégico de um serviço de saúde, pois permite fazer uma análise e mapeamento do fluxo dos pacientes, além de uma reflexão sobre as possibilidades de acesso para as pessoas que buscam o serviço.
\end{abstract}

Descritores: Instituições Acadêmicas. Assistência Odontológica. Triagem. Epidemiologia. 


\section{INTRODUÇÃO}

As condições de vida e de trabalho dos brasileiros são profundamente afetadas por diferenças socioeconômicas, e têm importante influência sobre os padrões epidemiológicos das doenças, que possuem associação com questões de vulnerabilidade social. Apesar de muitas melhorias terem sido implementadas nas últimas décadas no que tange à Saúde Bucal, esse setor ainda pode ser considerado um problema de Saúde Pública. As principais doenças e agravos em Saúde Bucal, tais como cárie, perda de dentes, problemas na adaptação das próteses, fístulas e abscessos, se configuram como problemas sociais e econômicos, uma vez que podem interferir na psique, bem como podem dificultar a inserção de indivíduos no mercado de trabalho. Observa-se que esta situação atinge especialmente as pessoas que vivem em precárias condições econômicas ${ }^{1}$.

Segundo a Federação Dentária Mundial (FDI, World Dental Federation) ${ }^{2}$ a saúde bucal é multifacetada e inclui, sem limitações, a capacidade de falar, sorrir, cheirar, saborear, tocar, mastigar, engolir e transmitir uma variedade de emoções, por meio de expressões faciais, com confiança, sem dor ou desconforto e na ausência de doença do complexo craniofacial.

As cidades que apresentam maiores problemas na capacidade de disponibilizar para sua população serviços de educação, saneamento, moradia, emprego e saúde, de maneira igualitária e satisfatória, são as que apresentam maiores índices de cárie e perda dentária; sendo assim, fica evidente que as condições socioeconômicas representam um fator de vulnerabilidade às doenças que atingem a cavidade oral, e que para promover saúde é necessário entender que os indivíduos são permeados por fatores sociais, ambientais, culturais e psicológicos que interferem no processo saúdedoença-cuidado, e a partir dessa compreensão, desenvolver estratégias que visem concatenar o máximo desses fatores no estabelecimento de ações mais efetivas em saúde, tanto na assistência quanto na promoção e prevenção ${ }^{1}$

Entre os indivíduos em idade adulta, há ainda um baixo índice de procura aos serviços odontológicos, apesar da diminuição do número de novos casos da doença cárie verificada nos últimos levantamentos nacionais. ${ }^{3}$ Existem então algumas lacunas sobre as quais imagina-se que sejam as razões para esta situação, como, por exemplo, baixa situação socioeconômica, baixo grau de escolaridade e a autopercepção de saúde bucal ruim, fazendo com que as pessoas não tenham poder aquisitivo e empoderamento suficiente para buscar sua saúde bucal. Ainda, com relação à população adulta, observa-se que entre as mulheres há uma maior procura por atendimento odontológico $^{3 .}$

Para se conhecer a situação dos serviços prestados, bem como das reais necessidades de uma população e sua caracterização, são necessários estudos descritivos para elaboração de hipóteses e resoluções visando planejamento em saúde e ensino, baseado no conhecimento epidemiológico da população de interesse. Dessa maneira, avalia-se a efetividade dos serviços prestados à população, bem como a resolubilidade e qualidade das estruturas e processos envolvidos. Assim, conhecer as necessidades terapêuticas dos usuários que procuram as clínicas de ensino pode prover subsídios para qualificar a atenção odontológica prestada nessas instituições, bem como o processo educativo e pedagógico desenvolvido ${ }^{4}$.

A construção da cidadania nos cursos de Odontologia, com comprometimento em relação a integração à realidade social e epidemiológica, passa por práticas educativas na área da saúde de maneira humanizada, formando profissionais contextualizados à realidade e buscando transformações nos locais em que estão inseridos. $\mathrm{O}$ atendimento à população deve ser visto com integralidade, discernindo seu papel social da 
técnica, compreendendo o todo, desde a estrutura organizacional, administrativa, funcional dos serviços e da instituição até o conhecimento da comunidade em que o paciente está inserido e suas necessidades sociais, indo ao encontro das Diretrizes Curriculares Nacionais dos cursos de graduação em Odontologia ${ }^{5}$.

O que se espera é que as universidades e os serviços de formação em Odontologia atuem de forma conjunta, se corresponsabilizando pela formação dos futuros profissionais, de forma que se tenha continuidade na construção do processo ensino/aprendizagem, com atuação na sua comunidade, visando aumentar a interação social e melhorar os serviços prestados. Por isso, vê-se a importância em avaliar as instituições formadoras de futuros profissionais nesta área para transpor os obstáculos e qualificar o ensino no contexto das necessidades de saúde da população ${ }^{6}$.

Portanto, o objetivo deste estudo foi analisar os encaminhamentos realizados pelo serviço de Triagem do curso de Odontologia da Universidade Federal de Santa Maria (UFSM), verificando quantos dos pacientes triados receberam algum atendimento nas clínicas do curso, ou seja, tiveram acesso a tratamento, e identificar as necessidades terapêuticas dos pacientes, por meio dos encaminhamentos que receberam da Triagem.

\section{METODOLOGIA}

\section{Delineamento do Estudo e Participantes}

Trata-se de um estudo observacional descritivo, de caráter quantitativo, e a sua execução foi aprovada por Comitê de Ética em Pesquisa da UFSM (CAAE 54136716.0.0000 .5346).

Utilizou-se dados secundários existentes nos registros das listas de espera do Serviço de Triagem do curso de Odontologia da UFSM e nos dados do software usado para registro de pacientes e procedimentos, denominado Gerenciador Odontológico. Foram incluídos no estudo os pacientes da lista de espera entre os anos de 2015 e 2017, sendo excluídos registros com dados incompletos ou ilegíveis.

Foi realizada análise documental dos encaminhamentos efetuados pelo setor de triagem. Para composição da amostra, foram considerados os encaminhamentos de pacientes adultos a cada semestre, e não o número absoluto de pacientes triados (considerando que alguns pacientes podem ter sido triados mais de uma vez ao longo do recorte de tempo analisado). A coleta de dados se deu no ano de 2018.

\section{Cenário}

O Curso de Odontologia da UFSM possui atualmente dezoito clínicas, onde os alunos da graduação realizam procedimentos de diversas especialidades e/ou complexidades. A indicação para cada clínica é feita pelo setor de Triagem do curso, porém existem outras vias de acesso como: i) encaminhamento direto de uma clínica para outra; ii) contato direto entre alunos/professores de diferentes semestres; iii) encaminhamento via cursos de pós-graduação da mesma instituição, cujos projetos efetuam levantamentos epidemiológicos e buscam uma solução para as necessidades das pessoas avaliadas. $\mathrm{O}$ indivíduo adulto precisa agendar uma avaliação no setor de Triagem, onde serão conduzidos exames clínicos com uso de espátula de madeira e, se necessário, exames radiológicos, para assim serem encaminhados para as clínicas mais adequadas para cada caso. Uma vez encaminhados, os pacientes são então alocados em uma lista de espera e têm suas consultas agendadas via telefone à medida que as vagas são disponibilizadas pelas clínicas.

As clínicas que realizam atendimento pediátrico/infantil possuem sua própria forma de captação de pacientes, bem como a clínica extramuros da Escola Estadual Irmão José Otão. Outra peculiaridade está relacionada à Clínica de Estomatologia, responsável pela avaliação de 
lesões e outras patologias de tecido mole, que recebe os pacientes diretamente, atendendo-os por ordem de chegada. Por essa razão, tais clínicas não foram incluídas neste estudo, já que não integram a lista de espera da Triagem.

Os pacientes que são atendidos nas clínicas do curso de Odontologia da UFSM são cadastrados em um sistema de informações interno, o Gerenciador Odontológico, que se trata de um software que registra, além de dados cadastrais dos pacientes, os procedimentos realizados pelos alunos nas clínicas. Esse sistema foi inicialmente utilizado pelas disciplinas de Dentística e pelas Clínicas Integradas (2007). A partir de 2014, houve uma mudança no software, quando foi expandido para outras clínicas. No ano de 2016 todas as clínicas passaram a ter suas atividades registradas no Gerenciador Odontológico.

Os procedimentos executados pelos alunos nas Clínicas são registrados em fichas próprias (não padronizadas e arquivadas em pastas destinadas a cada aluno/dupla). Posteriormente, funcionários da UFSM e bolsistas da Coordenação do Curso efetuam a transferência das informações para o Gerenciador Odontológico. Cada procedimento possui um código identificador, associado ao número de matrícula do aluno que realizou o atendimento e a data do mesmo. No cadastro do paciente, também são incluídos dados como data de nascimento, sexo e endereço.

\section{Extração e fonte dos dados}

As informações presentes nas listas de espera (nome, sexo, telefone e clínica para a qual o paciente foi encaminhado) foram coletadas, tabuladas e organizadas no software Microsoft Excel (2010) de acordo com o semestre e com as clínicas para as quais os pacientes receberam encaminhamento. Os dados do Gerenciador Odontológico foram coletados por meio de listas geradas pelo próprio software, contendo nome dos pacientes, sexo, data de nascimento, clínica em que foi atendido e data do atendimento. De posse dessas informações, foi feito um cruzamento dos dados para que fosse possível identificar os pacientes da lista de espera que faziam parte da lista de pacientes atendidos gerada pelo Gerenciador Odontológico.

\section{Variáveis}

Este estudo analisou os encaminhamentos feitos pelo serviço de Triagem, verificando se os pacientes tiveram ou não acesso ao atendimento. Para avaliação, a variável "atendimento pós Triagem" foi criada e aqueles que tiveram pelo menos um atendimento registrado no Gerenciador Odontológico, em todas as clínicas para as quais receberam encaminhamento, foram classificados na categoria "acesso". O paciente que recebeu encaminhamento para mais de uma clínica e teve algum atendimento registrado, mas não para todas as clínicas encaminhadas, foi considerado como alguém que teve "acesso parcial" ao atendimento. Já aquela pessoa que não teve nenhum registro encontrado no Gerenciador Odontológico foi considerada como alguém na categoria "sem acesso" ao atendimento. Além disso, foram analisadas quais clínicas receberam maior número de indicações, sendo possível descrever um perfil das necessidades odontológicas dos indivíduos avaliados na Triagem, já que cada clínica está relacionada a uma ou mais especialidades e a diferentes níveis de complexidade. Não foi analisado, neste estudo, se o tratamento foi concluído ou não.

Por meio do registro das datas de Triagem (lista de espera) e das datas de atendimento (Gerenciador Odontológico), foi possível verificar o tempo de espera dos pacientes que tiveram acesso ou acesso parcial ao atendimento nas clínicas do curso e esta variável foi classificada em três esferas: (1) atendidos no mesmo ano da Triagem; (2) atendidos após um ano de espera; e (3) atendidos após dois anos de espera. Também foi levantada a diferença da procura por atendimento 
relacionada ao sexo (feminino/masculino) e a faixa etária média dos usuários, porém apenas das pessoas que procuraram a Triagem e tiveram acesso; para aqueles que foram classificados como sem acesso, não foi possível levantar tal dado, pois a informação da data de nascimento não constava no Gerenciador Odontológico.

Finalmente, foi criada a variável de período em que o paciente buscou o serviço da Triagem, sendo categorizada de acordo com os semestres letivos, como segue: $1^{\circ}$ semestre de 2015; $2^{\circ}$ semestre de 2015; $1^{\circ}$ semestre de 2016; $2^{\circ}$ semestre de 2016; $1^{\circ}$ semestre de 2017; $2^{\circ}$ semestre de 2017.

\section{Análise}

Os dados coletados foram tabulados no software Microsoft Excel versão 2010 (Pacote
Office, Microsoft Corporation, USA), e posteriormente transferidos e analisados de forma descritiva utilizando o programa estatístico STATA 14.0 (Stata Corporation, College Station, TX, USA). Frequências absolutas e relativas das variáveis foram geradas para descrição do perfil das triagens e atendimentos realizados nas clínicas odontológicas.

\section{RESULTADOS}

O número total de encaminhamentos realizados, nos anos de 2015 a 2017, foi 3.945. O número de encaminhamentos de pacientes do sexo feminino $(64,8 \%)$ foi maior em relação ao sexo masculino $(35,2 \%)$, e a média de idade dos pacientes que tiveram acesso às clínicas do curso foi 51,2 anos (desvio padrão de 15,2) (tabela 1).

Tabela 1. Descrição do perfil das triagens e atendimentos realizados nas clínicas odontológicas da UFSM dos anos de 2015 a 2017

\begin{tabular}{lc}
\multicolumn{1}{c}{ Variável } & $\mathrm{n}(\%)$ \\
\hline Sexo & \\
$\quad$ Masculino & $1388(35,2)$ \\
$\quad$ Feminino & $2557(64,8)$ \\
Idade & $51,2(15,2)^{*}$ \\
Quando procurou Triagem & \\
$1^{\circ}$ Semestre 2015 & $1093(27,7)$ \\
$2^{\circ}$ Semestre 2015 & $263(6,7)$ \\
$1^{\circ}$ Semestre 2016 & $539(13,6)$ \\
$2^{\circ}$ Semestre 2016 & $572(14,5)$ \\
$1^{\circ}$ Semestre 2017 & $769(19,5)$ \\
$2^{\circ}$ Semestre 2017 & $713(18,0)$ \\
Atendimento após Triagem & \\
Não foi atendido (Sem Acesso) & $2377(60,2)$ \\
Foi atendido em pelo menos uma clínica (Acesso Parcial) & $590(14,9)$ \\
Foi atendido em todos os encaminhamentos (Acesso) & $982(24,9)$ \\
Tempo para o primeiro atendimento & \\
Atendido no mesmo ano & $1221(77,6)$ \\
Atendido após 1 ano de espera & $347(22,1)$ \\
Atendido após 2 anos de espera & $5(0,3)$ \\
\hline
\end{tabular}

* Média e desvio padrão de idade. Mínimo (14,1 anos) e máximo (92,6 anos). A variável idade apresenta dados faltantes (número válido $=2111$ ).

Com relação à distribuição do número de encaminhamentos realizados ao longo do período analisado, os dados demonstraram que o primeiro semestre de 2015 teve o maior número de encaminhamentos $(27,7 \%)$, seguido pelo primeiro e segundo semestres de 2017 (19,5\% e 18\%, 
respectivamente). $\mathrm{O}$ semestre com $\mathrm{o}$ menor número de encaminhamentos foi o segundo de 2015 (6,7\%). Do total, aproximadamente 40\% dos encaminhamentos resultaram em pelo menos um atendimento nas clínicas para as quais os pacientes foram encaminhados (tabela 1).

Os pacientes considerados com acesso representaram 24,9\% da amostra. Aqueles com acesso parcial e os encaminhamentos sem acesso representaram 14,9\% e $60 \%$ da amostra, respectivamente. Em relação ao tempo de espera para atendimento, foi observado que a maioria dos pacientes com acesso foram atendidos no mesmo ano em que fizeram a Triagem $(77,6 \%)$ (tabela 1$)$.

De acordo com a tabela 2 , a clínica que recebeu o maior número de encaminhamentos em relação ao total de encaminhamentos foi a
Clínica de Periodontia I (10,8\%), seguida pelas clínicas: Integrada I $(9,4 \%)$, Cirurgia III $(9,4 \%)$, Integrada III $(9,0 \%)$, Dentística II $(8,9 \%)$ e Periodontia II $(8,7 \%)$. As clínicas com menor número de encaminhamentos foram: Cirurgia II $(1,8 \%)$, Cirurgia IV $(2,7 \%)$ e Prótese Total $(4,4 \%)$. Dentre as clínicas que integram a amostra, e considerando o número de atendimentos em relação ao total de encaminhamentos, observou-se que a clínica de Dentística foi a que apresentou maior número de acessos (16,2\%), seguida pelas clínicas de Cirurgia III $(11,6 \%)$, Periodontia I $(9,9 \%)$ e Integrada I $(8,2 \%)$. As clínicas que apresentaram menor número de acessos em relação ao total de encaminhados foram: Cirurgia IV $(1,8 \%)$, Prótese Total $(1,8 \%)$ e Prótese Parcial Removível (2,5\%).

Tabela 2. Descrição dos encaminhamentos e atendimentos em relação ao total de pacientes e percentual de pacientes atendidos em cada clínica odontológica da UFSM dos anos de 2015 a 2017

\begin{tabular}{lccc}
\hline Clínica & $\begin{array}{c}\text { Encaminhamentos } \\
\text { da Triagem para } \\
\text { atendimento } \\
\mathbf{n}(\boldsymbol{\%})\end{array}$ & $\begin{array}{c}\text { Pacientes que } \\
\text { tiveram acesso/acesso } \\
\text { parcial ao } \\
\text { atendimento } \\
\mathbf{n}(\%)\end{array}$ & $\begin{array}{c}\text { Percentual de } \\
\text { pacientes } \\
\text { encaminhados que } \\
\text { receberam } \\
\text { atendimento }\end{array}$ \\
\hline Periodontia I & $661(10,8)$ & $242(9,9)$ & 36,6 \\
Clínica Integrada I & $579(9,4)$ & $201(8,2)$ & 34,7 \\
Cirurgia III & $570(9,4)$ & $284(11,6)$ & 49,8 \\
Clínica Integrada III & $553(9,0)$ & $119(4,8)$ & 21,5 \\
Dentística & $542(8,9)$ & $398(16,2)$ & 73,4 \\
Periodontia II & $532(8,7)$ & $200(8,1)$ & 37,6 \\
Prótese Parcial Removível & $481(7,8)$ & $62(2,5)$ & 12,9 \\
Clínica Integrada IV & $436(7,1)$ & $142(5,8)$ & 32,6 \\
Clínica Integrada II & $351(5,7)$ & $179(7,3)$ & 51,0 \\
Clínica Integrada V & $319(5,2)$ & $147(6,0)$ & 46,1 \\
Endodontia II & $300(4,9)$ & $139(5,7)$ & 46,3 \\
Endodontia I & $270(4,4)$ & $145(5,9)$ & 53,7 \\
Prótese Total & $259(4,2)$ & $44(1,8)$ & 17,0 \\
Cirurgia IV & $164(2,7)$ & $43(1,8)$ & 26,2 \\
Cirurgia II & $111(1,8)$ & $109(4,4)$ & 98,2 \\
\hline
\end{tabular}

Quando comparados o número de número de acessos que esta mesma clínica recebeu encaminhamentos feitos para cada clínica e o (tabela 2), verificou-se que as clínicas de Cirurgia 
II e de Dentística prestaram pelo menos um atendimento para 98,2\% e 73,4\%, respectivamente, dos encaminhamentos que receberam. Já as clínicas Periodontia I, Periodontia II, Integrada I e Integrada IV possibilitaram o acesso de menos de $40 \%$ dos encaminhamentos que tiveram. Em torno de $50 \%$ dos pacientes encaminhados para as clínicas de Integrada II, Integrada V, Endodontia I e II e Cirurgia III receberam pelo menos um atendimento. As Clínicas de Prótese Parcial Removível e Prótese Total atenderam 12,9\% e $17 \%$, respectivamente, dos encaminhamentos que receberam.

\section{DISCUSSÃO}

Este estudo analisou os encaminhamentos realizados pelo serviço de Triagem do curso de Odontologia da UFSM, identificando quais as necessidades terapêuticas dos pacientes e verificando quantos dos pacientes triados receberam algum atendimento nas clínicas (acesso a tratamento). O número total de encaminhamentos realizados, de 2015 a 2017 foi de 3.945, dos quais $60,2 \%$ não obtiveram o acesso ao atendimento nas clínicas do curso. A maioria dos encaminhamentos foram para indivíduos do gênero feminino, sendo de 51,2 anos a média de idade dos que tiveram acesso. As clínicas que receberam o maior número de encaminhamentos foram as de atendimento integrado, indicando que os indivíduos que procuram o serviço possuem um acúmulo de necessidades terapêuticas.

O acesso aos serviços prestados se dá por meio da demanda espontânea, ou seja, as pessoas interessadas no atendimento agendam uma avaliação no setor de Triagem ao início de cada semestre. Durante esta avaliação, os pacientes são examinados e encaminhados para a(s) clínica(s) mais adequada(s) à sua necessidade de tratamento. Esta avaliação é feita por cirurgiões-dentistas supervisores e alunos de graduação, que diagnosticam as necessidades identificadas no exame clínico e radiográfico (quando necessário), tomam nota dos dados do paciente e das clínicas para as quais ele será encaminhado, ocorrendo o acesso da população de forma semelhante às Instituições Federais de Ensino Superior (IFES) com curso de graduação em Odontologia da Região Sul do Brasil ${ }^{7}$. São essas as informações que compõem a lista de espera das clínicas da UFSM e os dados deste estudo.

As pessoas que procuram atendimento nas clínicas odontológicas da UFSM podem residir em qualquer área da cidade e, inclusive em outros municípios, pois não existe um território definido para o serviço. Além disso, o serviço ofertado não integra o Sistema Único de Saúde, e desta forma são cobradas taxas por consultas, procedimentos e/ou serviços externos (como, por exemplo, os serviços prestados por laboratórios de prótese dentária ou exames histopatológicos); acredita-se que tal cobrança possa limitar o acesso de muitos pacientes que buscam esse atendimento.

O serviço da Triagem do curso é destinado para indivíduos adultos, o que explica a faixa etária elevada encontrada nesse estudo, sendo a maioria dos usuários do sexo feminino. Junior e Souza (2011) também observaram que a prevalência do sexo feminino é maior quanto à procura dos serviços odontológicos, talvez em função da autopercepção do processo saúde-doença, bem como da maior disponibilidade de tempo relacionada à inserção informal da mulher no mercado de trabalho ${ }^{8}$.

$\mathrm{Na}$ fase de Triagem, as pessoas são esclarecidas sobre a não garantia de vaga para atendimento nas clínicas para as quais foram encaminhados. Atualmente, após o preenchimento das vagas disponíveis, aquelas pessoas que não foram agendadas são mantidas na lista de espera por um ano; após esse período de tempo, devem procurar novamente o serviço de Triagem. Esta definição de tempo de validade da Triagem foi feita a partir de 2017, pois foi verificado que alguns 
pacientes passavam longos períodos nas listas de espera e, não raro, quando eram agendados, as necessidades de tratamento já haviam sofrido algum tipo de alteração, não se enquadrando mais para a clínica do encaminhamento inicial.

A variação observada no número de encaminhamentos de um semestre para o outro no ano de 2015 se deve ao fechamento da lista de espera das clínicas integradas no segundo semestre, tendo em vista o excesso de encaminhamentos/triagens realizados no primeiro semestre daquele ano. Guimarães e Mello (2017) também descreveram como um problema, nas IFES analisadas por eles, as questões de reencaminhamentos para os serviços de captação de pacientes, além dos longos períodos de espera para atendimento ${ }^{7}$. A redução no número de encaminhamentos nos anos subsequentes a 2015 ocorreu devido a uma limitação no número de vagas para a Triagem, que teve o objetivo de reduzir a fila de espera.

Esta lista de espera não é organizada de acordo com risco ou urgência, mas sim com ordem cronológica de atendimento dos pacientes na Triagem, diferente do que se preconiza idealmente, que seria através da classificação de risco. A classificação de risco é uma ótima ferramenta para atender a população com maior equidade $\mathrm{e}$ organização do serviço ${ }^{9}$. Na estratificação de risco deve-se levar em consideração fatores biológicos, odontológicos, comportamentais

$\mathrm{e}$
socioeconômicos para se planejar o tratamento do paciente, bem como considerar a estratificação dessa população e identificar seus riscos e vulnerabilidades ${ }^{10}$.

Da mesma forma como foi constatado por Guimarães e Mello (2017), várias situações podem fazer com que o paciente da fila de espera não seja atendido, como por exemplo: alterações ou erros no registro das informações da lista de espera, problemas de telefonia, o horário de atendimento na clínica incompatível com a rotina do interessado, tratamento já executado em outro lugar ou, ainda, a clínica não considerar o paciente com o perfil de necessidades terapêuticas adequado ao que é proposto naquela disciplina ${ }^{7}$. Na Universidade Federal de Goiás (UFG), em um estudo realizado por Ferreira e colaboradores (2012) para avaliar o processo ensinoaprendizagem, bem como o funcionamento das clínicas, alunos do curso de Odontologia relataram que entre as maiores dificuldades estariam os problemas vinculados à organização no interior das IES, como horários, burocracias, número insuficiente de professores por clínica, falta de informatização, falta de pacientes e falta de materiais, impactando na formação final ${ }^{11}$.

Mais de $60 \%$ dos encaminhamentos realizados pela Triagem, ao longo do período analisado, não foram absorvidos pelas clínicas do curso de Odontologia da UFSM, o que significa que as pessoas não tiveram nenhuma das suas necessidades de saúde bucal assistidas pela instituição. Mesmo que a finalidade dos atendimentos realizados pelas clínicas do curso seja a formação profissional e que, neste sentido, possam existir alguns quesitos que devam ser preenchidos com relação à atividade clínica, é importante perceber que as pessoas que buscam atendimento possuem necessidades de saúde e devem ser tratadas com dignidade e respeito, e não como meios para a aquisição de habilidade de alguma técnica. Além disso, quando se organiza o atendimento baseado no tipo de procedimento/necessidade, se perde a noção de integralidade e de promoção de saúde - trata-se a boca, e não o indivíduo. A valorização da técnica em detrimento dos aspectos humanos leva à formação de profissionais pouco reflexivos, desatentos às necessidades da comunidade que procura o seu atendimento e pouco comprometimento com os aspectos sociais que cercam a prevenção de doenças e a promoção de saúde $^{12}$. 
As Diretrizes Curriculares Nacionais do Curso de Graduação em Odontologia definem que o perfil do profissional egresso deva ser pautado não apenas pelo rigor técnico e científico, mas também na capacitação para trabalhar com a saúde bucal de populações, levando em consideração aspectos socioeconômicos e culturais, agindo de maneira crítica e reflexiva e buscando transformar a realidade local de saúde. A formação do Cirurgião-Dentista deve ter como objetivo, além da prevenção, proteção e reabilitação da saúde, a prática integral e continuada da assistência, levando em conta que "a atenção à saúde não se encerra com $o$ ato técnico, mas sim, com a resolução do problema de saúde, tanto em nível individual quanto coletivo"s.

Um fato a ser destacado como ponto positivo é que o atendimento ocorreu no mesmo ano da Triagem para a maioria dos pacientes que receberam atendimento, já que o tempo de espera maior poderia agravar os problemas e demandas bucais desses indivíduos.

A grande maioria dos encaminhamentos foram para clínicas de atendimento integrado, doença periodontal, procedimentos cirúrgicos (especialmente exodontia) e necessidades protéticas, indicando que o indivíduo que procura o curso possui um acúmulo de necessidades terapêuticas, com grande número de perdas dentárias, necessitando tratamentos curativos, reabilitadores e de ações de redução de danos, ou seja, são desassistidos em relação à promoção e à prevenção em saúde. Clínicas como Dentística e Cirurgia absorvem mais rapidamente os encaminhamentos, pois nelas são realizados a maioria dos procedimentos que demandam menor tempo de atendimento. Verifica-se também que a população que procura o atendimento na UFSM é composta principalmente por adultos e idosos, fato que corrobora o acúmulo dos agravos bucais, um reflexo da falta ou precariedade dos cuidados com a saúde bucal ao longo da vida, e também dos fatores socioeconômicos e dos níveis de educação em saúde da população, frutos de um modelo de saúde curativista ${ }^{13}$.

A diminuição gradual no número de encaminhamentos para as disciplinas de Clínica Integrada IV e V e para a Cirurgia IV ocorreram devido à alteração curricular pela qual o curso passou recentemente, onde essas disciplinas foram extintas, dando lugar a uma carga horária maior para estágios em serviços de saúde. Conforme informações obtidas com a Coordenação do curso, as demais clínicas integradas tiveram como alteração a adição de outros procedimentos aos seus cronogramas, por exemplo: a Clínica integrada I passou a realizar tratamentos endodônticos, a Clínica Integrada II foi destinada a confecção de prótese fixa e a Clínica Integrada III recebe pacientes com múltiplas necessidades terapêuticas e protéticas. Além das Clínicas Integradas II e III, as únicas clínicas que também confeccionam próteses são a Prótese Total e a Prótese Parcial Removível. As disciplinas de Dentística II e III foram identificadas, neste trabalho, como uma única disciplina, porque durante a atualização do software Gerenciador Odontológico os dados anteriores ao segundo semestre de 2017 foram registrados com o mesmo nome. As demais clínicas atuam nas especialidades que as nomeiam e com grau crescente de dificuldade/complexidade.

$\mathrm{O}$ presente estudo tem como principais elementos limitadores o recorte de tempo e a atualização do programa Gerenciador Odontológico (ocorrida em 2014), que ocorreu de maneira gradual. Além disso, neste estudo não foi verificado se os tratamentos iniciados foram concluídos, tendo em vista que, para tanto, seria necessário acesso aos prontuários e planos de tratamento, e que como essas informações ainda não são unificadas, demandaria mais tempo. Por fim, como se trata de um estudo observacional descritivo, não há elementos para comparação dos 
dados analisados.

\section{CONCLUSÕES}

A constatação de que mais de $60 \%$ dos encaminhamentos realizados pela Triagem, de 2015 a 2017, não foram absorvidos pelas clínicas do curso de Odontologia da UFSM, traz à tona a necessidade de discussão sobre a estruturação das clínicas e sobre o próprio processo de Triagem, além da formação do profissional de saúde. Esse tipo de avaliação é fundamental para o planejamento estratégico de um serviço de saúde, pois permite fazer uma análise e mapeamento do fluxo dos pacientes triados no curso e permite uma reflexão sobre as alternativas de acesso para as pessoas que buscam o serviço. Tendo em vista que a integração ensino-serviço-comunidade é fundamental na formação de um profissional da saúde, este tipo de estudo permite começar uma reflexão sobre como estamos interagindo com a comunidade que procura atendimento em um serviço de uma instituição de ensino e como estruturar as clínicas e a forma de acesso a elas para que se satisfaça a demanda dos pacientes e as necessidades de aprendizagem dos estudantes.

\section{ABSTRACT}

Analysis of referrals determined by the screening processes in relation to the access to the clinics in the School of Dentistry at the Federal University of Santa Maria

The objective of this study was to describe the access to treatment and therapeutic needs of patients who seek care in the clinics of the School of Dentistry at the Federal University of Santa Maria (Brazil) by analyzing the referrals made by the school Screening Service, as well as verify how many of these patients effectively received dental care. It is a quantitative descriptive observational study, based on secondary data from the records of waiting lists of the school Screening Service and the data from the Dental Manager software used to register the procedures. Data from patients who sought dental services between 2015 and 2017 were included. Data were analyzed by using the statistical software STATA 14.0, showing relative and absolute frequencies. The total number of referrals made in the period was 3,945 , most of which to female patients at the average age of 51.2 years. From the total of referrals, in $60.2 \%(2,377)$ of cases the service was not completed. From the situations which resulted in dental care service, in $77.6 \%(1,221)$ of the cases, the service was not provided during the same year. Most referrals were sent to the integrated care clinics, what indicates an assortment of therapeutic needs. This kind of evaluation is fundamental for the strategic planning of a health service, since it provides an analysis and mapping of patient influx in addition to reflecting upon the possibilities of access to people who seek the service.

Descriptors: Academic Institutions. Dental Assistance. Screening. Epidemiology.

\section{REFERÊNCIAS}

1. Silva JV, Machado FCA, Ferreira MAF. As desigualdades sociais e a saúde bucal nas capitais brasileiras. Ciênc Saúde Colet. 2015;20(8):2539-48.

2. Glick M, Williams DM, Kleinman D V., Vujicic M, Watt RG, Weyant RJ. A new definition for oral health developed by the FDI World Dental Federation opens the door to a universal definition of oral health. Int Dent J. 2016 Dec;66(6):322-4.

3. Araújo CS, Lima RC, Peres MA, Barros AJD. Utilização de serviços odontológicos e fatores associados: um estudo de base populacional no sul do Brasil. Cad Saúde Pública. 2009;25(5):1063-72.

4. Reis SCGB, Santos LB, Leles CR. Clínica integrada de ensino Odontológico: perfil dos usuários e necessidades odontológicas. ROBRAC. 2011;20(4):46-51.

5. Brasil. Ministério de Educação. Conselho Nacional de Educação. Câmara de Educação 
Superior. Resolução CNE/CES 3, de 19 de fevereiro de 2002. Institui Diretrizes Curriculares Nacionais do Curso de Graduação em Odontologia. Diário Oficial da União, 2002; 04 mar.

6. Faé JM, Junior MFS, Carvalho RB, Esposti CDD, Pacheco KTS. A integração ensinoserviço em Odontologia no Brasil. Rev ABENO. 2016;16(3):7-18.

7. Guimarães FAF, Mello ALSF. Prestação de serviços odontológicos em instituições federais públicas de ensino superior e a integração com a rede de atenção à saúde. Rev ABENO. 2017;17(3):10-20.

8. Júnior ECS, Souza TB. Estudo da demanda ambulatorial da clínica de odontologia da Universidade do Estado do Amazonas. Ciênc Saúde Colet. 2011;16(Supl. 1):993-7.

9. Neto JP, Mendes KLC, Wada RS, Sousa MLR. Relação entre classificações de risco utilizadas para organização da demanda em saúde bucal em município de pequeno porte de São Paulo, Brasil. Ciênc Saúde Colet. 2017;22(6):1905-12.

10. Schiller CA, Paciornik DKF, Afonso GP, Graziani G, Kriger L. Linha guia de saúde bucal. Curitiba: Secretaria de Estado da Saúde do Paraná, 2014. 76 p. [Internet]. [acesso em 2018 Out 31]. Disponível em: http://www.saude.pr.gov.br/arquivos/File/L inha_Guia_Rede_de_Saude_Bucal.pdf.

11. Ferreira NP, Dantas TS, Sena-Filho M, Rocha DG. Clínica integrada e mudança curricular: desempenho clínico na perspectiva da integralidade. Pesqui Bras Odontopediatria Clín Integr. 2012;12(1):339.

12. Gonçalves ER, Verdi MIM. Os problemas éticos no atendimento a pacientes na clínica odontológica de ensino. Ciênc Saúde Colet. 2007;12(3):755-64.

13. Souza JGS, Souza SE, Sampaio AA, Silveira MF, Ferreira EF, Martins AMEBL. Autopercepção da necessidade de prótese dentária total entre idosos brasileiros desdentados. Ciênc Saúde Colet. 2016;21 (11):3407-15.

\section{Correspondência para:}

Jessye Melgarejo do Amaral Giordani

e-mail: jessyesm@hotmail.com

Avenida Roraima, 1000

Prédio 26F Bairro Camobi

97105-900 Santa Maria/RS 